\title{
Single shot medium dose melphalan in resistant/relapsed myeloma: a bridge to target therapies?
}

\author{
Cristina Clissa, Federica Loscocco, Alessandro Isidori, Sara Barulli, Lara Malerba, Barbara Guiducci and Giuseppe Visani* \\ Department of Hematology and Stem Cell Transplant Center AORMN Pesaro, Italy
}

\section{Case Study}

Multiple myeloma (MM) is a malignant plasma cell disorder with no curative therapy. In the past decade, proteasome inhibitors (PI) and immunomodulatory drugs (IMiDs) have led to significant improvement in the treatment of multiple myeloma [1,2]. However, almost all patients will ultimately relapse. Furthermore, patients refractory to proteasome inhibitors, IMIDs or both, have an extremely poor prognosis [1,2]. Within the last couple of years, second generation PI and IMIDs, deacetylase inhibitors and monoclonal antibodies were approved and are now commercially available. These drugs are not only a significant advancement and an appealing option for patients with refractory or relapsed (RR) MM, but also an expensive option that cannot be administered to all RRMM patients all over the world $[1,2]$.

With this in mind, we treated 12 RRMM patients with a single shot of medium dose melphalan $(60 \mathrm{mg} / \mathrm{m} 2)$, from October 2014 to December 2016. The median age was 72 years (range, $62-79$ ) and the median time from initial diagnosis until melphalan treatment was 51 months (range, 24 - 144). Patients were heavily pretreated with a median number of 3 prior lines of therapy. All patients were refractory to the previous therapeutic regimens, and had failed to respond or were refractory to bortezomib containing regimens. Ten out of 12 (84\%) patients had previously received at least one IMiD, 8/12 (67\%) autologous stem cell transplantation (ASCT) and 1 allogeneic stem cell transplantation. Seven out 12 patients were refractory to both Bortezomib and one IMID (Table 1). All patients were not eligible for any clinical trial available at the Institution; they signed informed consent prior to receiving treatment. All patients had cytopenia

Table 1. Characteristics of Patients. Note: "detected by standard karyotype

\begin{tabular}{|l|c|c|}
\hline \multicolumn{1}{|c|}{} & $\mathbf{N}$ & $\mathbf{\%}$ \\
\hline Median age (range) & $72(62-79)$ & \\
\hline Chromosomal abnormalities* & & 58 \\
\hline yes & 2 & 17 \\
\hline no & 3 & 25 \\
\hline not available & & \\
\hline ECOG - PS & 3 & 25 \\
\hline $0-1$ & 9 & 75 \\
\hline $2-3$ & 0 & \\
\hline Renal disfunction & $51(24-144)$ & \\
\hline Median time diagnosis-treatment (range, months) & & 17 \\
\hline $\mathbf{N}^{\circ}$ of prior lines of therapy & 2 & 83 \\
\hline$<3$ & 10 & 58 \\
\hline 3 or more & 7 & 67 \\
\hline Prior IMiDs & 8 & 8 \\
\hline Prior autologous SCT & 1 & \\
\hline Prior allogenic SCT & & \\
\hline
\end{tabular}

(anemia grade III, neutropenia grade III/IV, and thrombocytopenia grade IV). We observed 3 cases of gastrointestinal toxicity ( 1 bleeding grade II, 1 subocclusion grade II, 1 mucositis grade IV according to WHO), 3 cases of clinically documented infection (1 Escherichia coli bacteremia, 1 fever of unknown origin, 1 erysipela) and 2 deep vein thrombosis. All patients were hospitalized.

Response was assessed between six and eight weeks after melphalan therapy. Overall, 10 out of 12 patients had a response ( 1 complete response, 3 very good partial response, 2 partial response and 4 stable disease); only 2 had progressive disease. Median overall survival was 11 months (range, 2 -37).

Ten out 12 patients (84\%) relapsed after a median time of 5 months (range: 2-12). One additional patient died in partial response 8 months after therapy, due to other causes, and 1 patient is still alive, in complete remission 18 months after melphalan. He underwent ASCT on October 2015 ( 2 months after melphanam medium dose) and started maintenance therapy with lenalidomide (off label) on March 2016 (day +120 from ASCT). He stopped maintenance therapy on March 2017 due to recurrent infections. The patient is still in good clinical conditions, and in complete remission.

Our experience demonstrated the efficacy of single dose melphalan in inducing a significant rate of responses in patients with extremely poor prognosis. In this setting, recently Kumar, et al. [1] reported on more than 500 unselected patients who have received at least 3 prior lines of therapy, refractory to both an IMiDs and a PI, and had been exposed to an alkylating agent. This patient group is highly representative of those who are most in need of newer therapies. Median overall survival after treatment with different drugs or combo, such as Bendamustine, DT-PACE, high dose dexamethasone, etoposide, cyclophosphamide, etc, was 13 months. This is in line with our data, suggesting that single-shot medium dose melphalan could be a good option for highly resistant patients. In this view, patients refractory to proteasome inhibitors and IMiDs are probably still sensitive to alkylating agents and could be rescued with medium dose melphalan $[2,3]$. We suggest, therefore, melphalan as a "bridge" strategy for further therapy, particularly in patients needing immediate disease control. Within the last two years, several novel agents were approved for use as salvage therapy for RRMM: second-generation

${ }^{*}$ Correspondence to: Giuseppe Visani, MD, Department of Hematology and Stem Cell Transplant Center AORMN Pesaro, Via Cesare Lombroso 1 , 61121 PESARO, Italy, Tel: +39.0721.364039; Fax: +39.0721.364052; E-mail: pesarohematology@yahoo.it

Received: June 29, 2018; Accepted: July 16, 2018; Published: July 20, 2018 
IMiDs (pomalidomide), second-generation proteasome inhibitors (e.g., carfilzomib, ixazomib), deacetylase inhibitors (panobinostat), monoclonal antibodies (e.g., elotuzumab, daratumumab) [2,3]. These novel agents appear promising to improve outcome, but they represent a very expensive choice, not suitable in all countries worldwide. Moreover, recommendations on the management of RRMM are contradictory [4]: thus, no uniform treatment has yet been established for these patients. Nevertheless, RRMM is a highly heterogeneous disease, thus there is need for multiple therapeutic options, affordable also for countries with lower- and lower/middle-income economies. Therefore, relapse management requires an individual approach based on assessment of patient, disease and treatment-related factors. Frailty, comorbidity, disability, quality of life and the overall goals of care are primary considerations when selecting an appropriate treatment.

\section{Conclusion}

In conclusion, single shot medium dose melphalan could be an affordable and safe therapy, able to control aggressive relapse or to reduce disease burden prior to targeted therapy, even in this era in which several novel drugs are available.

\section{Acknowledgements}

Supported in part by AIL Pesaro Onlus.

\section{References}

1. Kumar SK, Dimopoulos MA, Kastritis E (2017) Natural history of relapsed myeloma, refractory to immunomodulatory drugs and proteasome inhibitors: a multicenter IMWG study. Leukemia 31: 2443-2448.

2. Cook G, Zweegman S, Mateos MV, Suzan F, Moreau P (2018) A question of class Treatment options for patients with relapsed and/or refractory multiple myeloma. Crit Rev Oncol Hematol 121: 74-89. [Crossref]

3. Chim CS, Kumar SK, Orlowski RZ, Cook G, Richardson PG, et al. (2018) Management of relapsed and refractory multiple myeloma: novel agents, antibodies, immunotherapies and beyond. Leukemia 32: 252-262.

4. Sonneveld P, De Wit E, Moreau P (2017) How have evaluations in strategies for the treatment of relapsed/refractory multiple myeloma translated into improved outcomes for patients? Crit Rev Oncol Hematol 112: 153-170.

Copyright: (C2018 Clissa C. This is an open-access article distributed under the terms of the Creative Commons Attribution License, which permits unrestricted use, distribution, and reproduction in any medium, provided the original author and source are credited. 